\title{
Extensive Ameloblastic Fibroma in an Adolescent Patient: A Case Report with a Follow-Up of 4 Years
}

\author{
Poramate Pitak-Arnnop a,b \\ André Chainec \\ Kittipong Dhanuthaid \\ Jacques-Charles Bertrand \\ Chloé Bertolus ${ }^{\dagger}$
}

\begin{abstract}
Ameloblastic fibroma (AF) is a rare odontogenic tumour of the jaw which usually occurs in the first 2 decades of life. The common clinical manifestation is a slow-growing swelling. We report a case of a 16-year-old male patient presenting with extensive AF of the mandible. He underwent a conservative enucleation. The tumour recurred 2 years after the initial surgery, requiring the second enucleation. The patient has continued to be followed closely and has been disease-free for 4 years. Recent evidence suggests that the recurrent rate of AF is relatively high, and malignant transformation of AF may occur after recurrences or multiple surgeries. A conservative treatment approach with close surveillance is recommended. Anatomical limitations should be taken into account, especially when enucleation of $A F$ in the posterior portion of the jaw is performed. Current surgical pathologic issues of the tumour are also discussed. (Eur J Dent 2009;3:224-228)
\end{abstract}

Key words: Ameloblastic fibroma; Jaw tumour; Odontogenic tumour.

- a PhD candidate and resident, Department of Oral, Craniomaxillofacial and Facial Plastic Surgery, Faculty of Medicine, University Hospital of Leipzig, Leipzig, Germany; Formerly, Department of Maxillofacial Surgery, AP-HP,

Pitié-Salpêtrière University Hospital,

Faculty of Medicine, University Paris 6 (Pierre et Marie Curie), Paris, France.

b PhD candidate, Laboratory of Medical Ethics and Legal Medicine, Faculty of Medicine, University Paris 5 (René Descartes), Paris, France.

c Consultant surgeon, Department of Maxillofacial Surgery, AP-HP, Pitié-Salpêtrière University Hospital, Faculty of Medicine, University Paris 6 (Pierre et Marie Curiel, Paris, France.

d Associate Professor, Department of Oral Pathology, Faculty of Dentistry, Chulalongkorn University, Bangkok, Thailand. e Head Professor and Chairman, Department of Maxillofacial Surgery, AP-HP, Pitié-Salpêtrière University Hospital, Faculty of Medicine, University Paris 6 (Pierre et Marie Curiel, Paris, France.

f Assistant Professor, Department of Maxillofacial Surgery, AP-HP, Pitié-Salpêtrière University Hospital, Faculty of Medicine, University Paris 6 (Pierre et Marie Curie), Paris, France.

Corresponding author: Dr. Poramate Pitak-Arnnop Klinik und Poliklinik für Mund-, Kiefer- und Plastische Gesichtschirurgie,

Universitätsklinikum Leipzig AöR, Nürnberger Str. 57, 04103 Leipzig, Deutschland

Phone: +493419721100

Fax: +493419721109

E-mail: poramate.pitakarnnoplagmail.com 


\section{INTRODUCTION}

Ameloblastic fibroma (AF) is an uncommon odontogenic ectomesenchymal tumour of the jaw which is frequently seen in the first 2 decades of life. Its incidence is approximately $1.5-4.5 \%$ of all jaw tumours. ${ }^{1-4}$ AF was first described by Krause in 1891.5 Up until now, 123 patients have been reported in the English literature. ${ }^{2}$

$A F$ is a benign, slow-growing tumour. Most of the cases are asymptomatic and discovered during routine oral examination as a unilocular radiolucent lesion. An enlarging lesion usually has a multilocular, radiolucent pattern. ${ }^{1-3,6}$ Histologically, AF is consisted of groups of an odontogenic epithelium in the dentalpapilla like background without dental hard tissue formation. ${ }^{1,2,6}$ It should be noted that AF bears resemblance to various odontogenic tumours, including ameloblastic fibrodentinoma, ameloblastic fibro-odontoma, and odontoma. ${ }^{1-3}$

Recent evidence suggests that AF exhibits a high recurrent rate and, in some cases, malignant transformation. ${ }^{2}$ This finding differs from the existing knowledge, arousing more attention on this tumour. The aim of this article was to report a case of a 16-year-old patient presenting with extensive AF of the mandible which developed within 5 years. Clinicopathologic considerations of the tumour were also discussed.

\section{CASE REPORT}

A 16-year-old white male was referred to our department for evaluation and management of a hard, painful mass of the left cheek. He had a progressive swelling on this area for approximately 4 months. His medical history was unremarkable.

Head and neck examination revealed no abnormality other than a considerable left facial swelling, and an expanded and indurate mandibular buccal vestibule. On palpation of the left mandibular angle, cortical perforation of both buccal and lingual cortices, pain and tenderness were found. The left lower third molar was clinically absent. All neighbouring teeth were vital with no evidence of other dental abnormalities.

A panoramic radiograph showed a multilocular radiolucent lesion with poorly-demarcated borders, involving the molar-ramus area of the left mandible. The mandibular canal was not invaded, but was displaced inferiorly. The left lower third molar was found at the inferior mandibular borders (Figure 1B). The patient's panoramic film taken at 5 years before this presentation demonstrated that the lower left third molar tooth germ was in a normal position (Figure 1A). Computed tomography (CT) illustrated a voluminous, spaceoccupying lesion at the posterior part of the left mandible. The tumour perforated both buccal and lingual cortices of the jaw (Figure 2).

Aspiration biopsy was negative, indicating a non-cystic lesion. Histological examination of the incisional biopsy tissue demonstrated strands, cords, and islands of odontogenic epithelium within immature myxoid mesenchymal tissue resembling the dental papilla (Figure 3). The definite diagnosis was AF.

The patient underwent an enucleation via an intra-oral vestibular approach with the preservation of the inferior dental nerve and the inferior and posterior mandibular border. The impacted third molar tooth within the lesion was removed at the time of surgery. The immediate post-operative period was uneventful.

The tumour relapsed 2 years after the initial surgery, requiring the second enucleation (Figure 1C). The patient has continued to be followed closely and has been disease-free for 4 years. His condition has remained satisfactory, including the facial growth, throughout the follow-up period.

The recommendations of the Helsinki declaration were thoroughly maintained during the study. Ethical approval of this article was exempted by the Committee of Human Subject Protection in Biomedical Research (Comité de Protection des Personnes: CPPJ of Paris and its suburb, but the patient did not allow us to publish his clinical pictures. The national guideline 'Déclaration de Commission Nationale de l'Informatique et des Libertés (CNIL)' was followed in order to protect patient confidentiality.

\section{DISCUSSION}

AF is an uncommon odontogenic tumour. In general, it does not present with dental hard tissues. If the lesion is similar to AF, but contains dentinoid tissue or dentin without or with enamel formation, it should be designated under the names 'ameloblastic fibrodentinoma' (AFD) and 'ameloblastic fibroodontoma' (AFO), respectively. ${ }^{1-3}$ The presentation of these different 
tumours may be due to the degree of inductive changes and dental hard tissue formation of the same neoplastic entity (the 'continuum' concept). However, this concept has been challenged due to a number of reasons. Several longstanding, residual or recurrent AFs have not demonstrated further maturation into a more differentiated lesion such as AFD, AFO, or odontoma. Moreover, AFs are encountered at ages beyond the completion of odontogenesis, and some AFs occur at the higher age than AFO. 7,8

It is believed that odontogenic epithelium in AF arises from the primordial enamel organ, and the stromal component originates from the dental papilla. ${ }^{9}$ The biological behaviour of AF shares the nature between harmatoma and true neoplasm. Clinically, this tumour is well-encapsulated and slow-growing. AF in the mandibular location, chiefly in the posterior mandible, is more common than in the maxillary counterpart. The patients usually present with a hard swelling, but intraoral ulceration, pain, tenderness, or drainage may also be observed. In cases of AF in a pericoronal location, the involved tooth may fail to erupt into the oral cavity (as seen in our patient). Radiographically, unilocular radiolucency with a smooth outline is associated with asymptomatic patients, while cases with jaw swelling generally have the multilocular, radiolucent pattern. Most of the AF lesions exhibit radiopaque borders. ${ }^{1-3,6}$

Once a patient presents with an extensive tumour as seen in our case, the differential diagnosis list should include ameloblastoma, odontogenic keratocyst and myxoma. It remains unknown when the tumour began in our patient. A progressive swelling seems to be only a noticeable symptom of an enlarging lesion. This addresses the importance of routine oral examination in childhood and adolescence which should be carried out thoroughly for both soft and

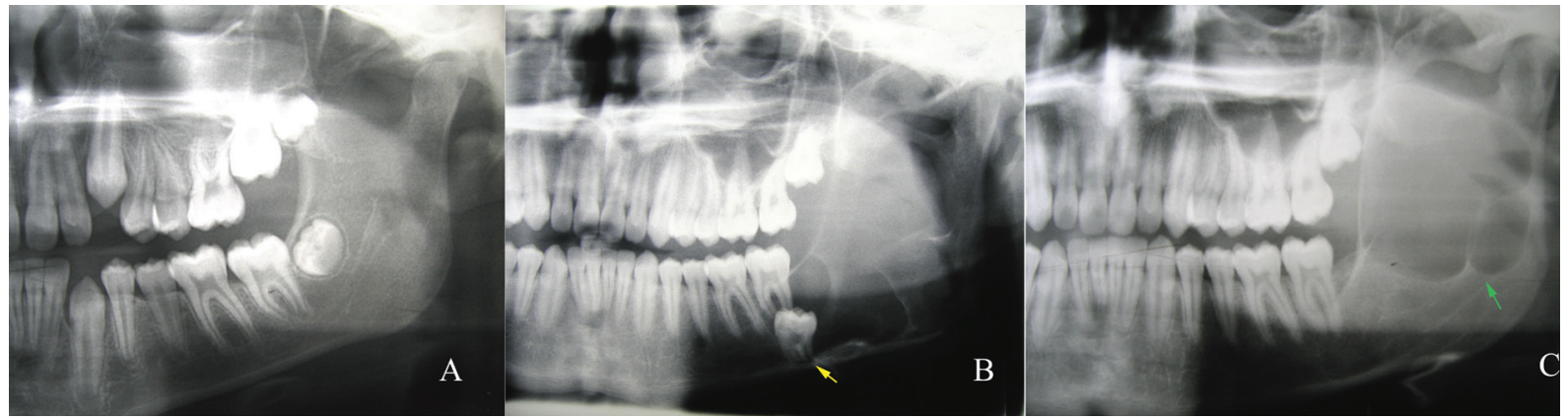

Figure 1. A part of panoramic radiograph showing: A) the mandible at 5 years before the presentation of the tumour; the lower left third molar tooth germ was in a normal position, B) a voluminous tumour involving the molar-ramus area of the left mandible and displacement of the left lower third molar (yellow arrow), and C) the recurrent tumour at the left mandibular ramus (green arrow).

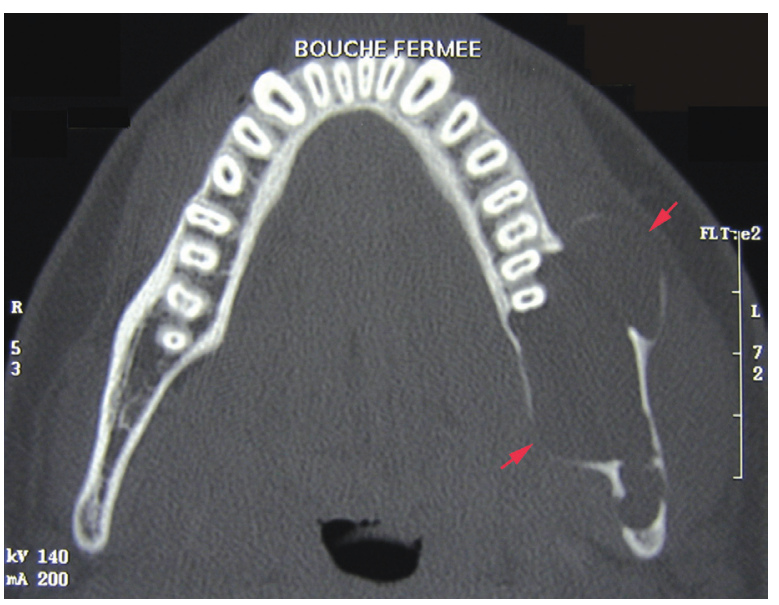

Figure 2. Non-contrast axial CT at bone algorithm showing an expansive, homogeneously hypoattenuated mass occupying the posterior part of the left mandible. Buccal and lingual cortical perforation was evident (red arrow).

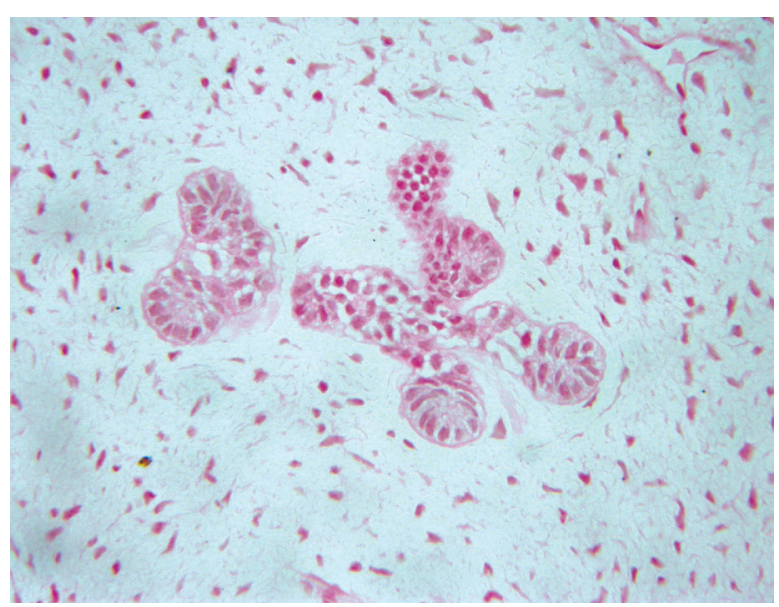

Figure 3. Photomicrograph showing cords and islands of odontogenic epithelium in a primitive connective tissue background resembling a dental papilla without the formation of dental hard tissues (Haematoxylin \& eosin stain, original magnification 40x). 
hard tissues of the oral cavity. Had the patient undergone routine check-up, the lesion would have been detected earlier before it developed into a voluminous size.

Microscopically, AF comprises strands and islands of an odontogenic epithelium in a loose and primitive connective tissue stroma which is characteristic of dental papilla lembryonic dental pulp). The odontogenic epithelial cells are similar to those of ameloblastoma. Tiny islands resembling the follicular stage of the developing enamel organ may be observed. ${ }^{1,2,6}$ Some recurrent cases developed dentin formation with or without enamel structures, and subsequently differentiate over time into odontoma. Of particular note, $A F$ in young populations may resemble the primitive stage of odontoma. ${ }^{2}$ In cases undergo malignant transformation, there is unequivocal changes in the mesenchymal component, and the odontogenic epithelium is completely disappeared. The diagnosis of malignant transformation of AF is established by tracing back the primary or recurrent lesions in which typical features of $A F$ could be recognised. ${ }^{2,3}$

The hybrid tumour between calcified odontogenic cyst (COC; Gorlin's cyst) and AF has been reported in the literature. Care must be taken to differentiate this mixed tumour from either AFO or AFD. The aetiology of the coexistence remains unclear. It is possible that odontogenic epithelium of $\mathrm{COC}$ induces the surrounding mesenchymal tissues to develop the other separate lesion..$^{10,11}$ However, Altini and Farman ${ }^{12}$ suggested that cystic degeneration of AF caused the development of the $\mathrm{COC}$ component. This collision phenomenon may also occur with other cystic lesions, such as dentigerous cyst or unicystic ameloblastoma. ${ }^{13}$

The name of $A F$ indicates a non-aggressive behaviour. However, a large series of AF revealed that its recurrent rate at 10 years after operation was approximately $70 \% .{ }^{2}$ Malignant transformation of $A F$ has been reported sporadically in recurrent AF or after multiple surgeries. ${ }^{37,14,15}$ Chen et al ${ }^{2}$ found that 14 of 41 recurrent AF cases developed malignant changes, and the estimated 10-year malignant transformation rate was one-fourth of the cases. Patient age at the presentation (> 22 years oldl was found to be the only potential risk of malignant AF. ${ }^{2}$ Therefore, based on evidence at present, close and long-term follow- up is indeed crucial. Special care should be taken in recurrent cases with regard to malignant transformation. However, these data must be interpreted with caution because malignant transformation of a benign tumour may be easily and frequently accepted for publication (so-called 'publication bias'), resulting in an exaggerated and overestimated incidence.

Malignant transformation of AF was found to be associated with oncogenic aberrations in tumour-related genes. ${ }^{9}$ Mesenchymal proliferation within the tumour resulting in a loss of an epithelial component, is a usual presentation of sarcomatous changes of AF. ${ }^{2}$ Hence, the histological distinction between ameloblastic fibrosarcoma arising from pre-existing $A F$ and conventional (true) ameloblastic fibrosarcoma seems to be difficult. Reviewing the previous histopathological slides when the tumour recurs is beneficial.,14,15 If malignant changes occur in both epithelial and stromal components, the tumour is dubbed 'ameloblastic carcinosarcoma'.? Regional lymphadenopathy and distant metastasis is infrequent in the vast majority of AF cases with malignant transformation. ${ }^{2}$

Management of AF varies between reports. In general, a conservative approach, such as enucleation with curettage of the surrounding bone, should be applied for young patients. Conversely, an extensive tumour and/or multiple recurrences necessitate more radical therapies. Recurrence commonly results from incomplete removal of the tumour. ${ }^{1-3}$ The recurrence in our patient may be due to a residual tumour following the conservative surgery. Limited surgical access in the posterior mandibular region complicates complete tumour removal.

CT scan is of clinical value during both the treatment planning and the surveillance of recurrence. Some authors suggested the use of proliferating indices, such as AgNOR, PCNA and $\mathrm{Ki}-67$, to institute an appropriate treatment for each patient, and possibly, to suggest the malignant development. ${ }^{2,15}$ However, there is still little evidence supporting this notion; thereby, more investigations are still warranted.

\section{CONCLUSIONS}

The patient reported here presented an uncommon feature of $\mathrm{AF}$ : a voluminous lesion 
occupying the posterior portion of the mandible. However, he was relatively young at the time of presentation. We, therefore, decided to apply a conservative approach and a 'wait and see' protocol for this patient.

Because of its high recurrent rate and a possibility of malignant transformation, the term 'benign tumour' in case of AF should not falsely impart a simultaneous designation of 'nonaggressive'. Complete excision with close followup is highly recommended.

\section{ACKNOWLEDGEMENTS}

The authors would like to thank Dr. Salima Bellefqih for her histological diagnosis.

This article was completed in partial fulfillment of the requirements for the 'Doctorat en sciences' (PhD/DSc) degree at the University Paris 5 (René Descartes) and the 'Doktor der Zahnheilkunde' (Dr.med.dent.) degree at the University of Leipzig by the first author.

\section{REFERENCES}

1. Slootweg PJ. Ameloblastic fibroma/fibrodentinoma. In: Barnes L, Evenson JW, Reichart P, Sidransky D, eds. The World Health Organization Classification of Tumours. Pathology and Genetics of Head and Neck Tumours. Lyon: IARC Press, 2005:308.

2. Chen Y, Wang JM, Li TJ. Ameloblastic fibroma: a review of published studies with special reference to its nature and biological behavior. Oral Oncol 2007;43:960-969.

3. Chen Y, Li TJ, Gao Y, Yu SF. Ameloblastic fibroma and related lesions: a clinicopathologic study with reference to their nature and interrelationship. J Oral Pathol Med 2005;34:588-595.

4. Cohen DM, Bhattacharyya I. Ameloblastic fibroma, ameloblastic fibro-odontoma, and odontoma. Oral Maxillofac Surg Clin North Am 2004;16:375-384.

5. Kruse A. Über die Entwicklung cystischer Geschwülste im Unterkiefer. Arch Path Anat 1891;124:137-148.

6. McGuinness NJ, Faughnan T, Bennani F, Connolly CE. Ameloblastic fibroma of the anterior maxilla presenting as a complication of tooth eruption: a case report. $J$ Orthod 2001;28:115-118.

7. Philipsen HP, Reichart PA, Praetorius F. Mixed odontogenic tumours and odontomas. Considerations on interrelationship. Review of the literature and presentation of 134 new cases of odontomas. Oral Oncol 1997;33:86-99.

8. Takeda Y. Ameloblastic fibroma and related lesions: current pathologic concept. Oral Oncol 1999;35:535-540.
9. DeLair D, Bejarano PA, Peleg M, El-Mofty SK. Ameloblastic carcinosarcoma of the mandible arising in ameloblastic fibroma: a case report and review of the literature. Oral Surg Oral Med Oral Pathol Oral Radiol Endod 2007;103:516520.

10. Lin CC, Chen CH, Lin LM, Chen YK, Wright JM, Kessler $\mathrm{HP}$, et al. Calcifying odontogenic cyst with ameloblastic fibroma: report of three cases. Oral Surg Oral Med Oral Pathol Oral Radiol Endod 2004;98:451-460.

11. Yoon JH, Kim HJ, Yook JI, Cha IH, Ellis GL, Kim J. Hybrid odontogenic tumor of calcifying odontogenic cyst and ameloblastic fibroma. Oral Surg Oral Med Oral Pathol Oral Radiol Endod 2004;98:80-84.

12. Altini M, Farman AG. The calcifying odontogenic cyst. Eight new cases and a review of the literature. Oral Surg Oral Med Oral Pathol 1975;40:751-759.

13. Usubütün A, Atayar C, Basal N, Araz K. Cystic ameloblastic fibroma. Br J Oral Maxillofac Surg 2002;40:512-514.

14. Williams MD, Hanna EY, El-Naggar AK. Anaplastic ameloblastic fibrosarcoma arising from recurrent ameloblastic fibroma: restricted molecular abnormalities of certain genes to the malignant transformation. Oral Surg Oral Med Oral Pathol Oral Radiol Endod 2007;104:72-75.

15. Kobayashi K, Murakami R, Fujii T, Hirano A. Malignant transformation of ameloblastic fibroma to ameloblastic fibrosarcoma: case report and review of the literature. $J$ Craniomaxillofac Surg 2005;33:352-355. 